\title{
(F)utilité des traitements en réanimation
}

\section{Is too much of a good thing a bad thing?}

\author{
A. Durocher \\ (C) SRLF et Lavoisier SAS 2015
}

Les termes " utilité » et " futilité » ne différent que d'une consonne et sont souvent présentés comme des contraires. Mais les définitions fournies par deux dictionnaires de la langue française mettent bien en évidence à la fois les différences entre futilité et inutilité, mais aussi les difficultés d'interprétation de ces termes en médecine.

\section{Définitions}

Futile : qui a peu de valeur, qui ne nécessite pas qu'on s'y arrête (Larousse) ;

qui est de peu de conséquences, de peu de valeur (Littré) ; utile : qui peut rendre service à quelqu'un, lui être profitable, lui procurer un avantage, qui a un rôle :

efficace, qui est nécessaire (Larousse) ;

qui sert à quelque chose (Littré).

Inutile : qui ne sert à rien, qui n'apporte rien, qui est superflu (Larousse) ;

qui n'est pas utile, qui ne rend pas de service, dont on ne se sert pas (Littré).

La SRLF, dans ses recommandations : « Limitation et arrêt des traitements en réanimation adulte " considère que le terme "futilité thérapeutique ", issu de la littérature anglosaxonne, imprécis et ambigu, doit être évité [1].

Cependant, la notion de futile care a émergé dans la littérature médicale, même s'il n'y a pas de définition médicale consensuelle de ce terme, car la notion de futilité fait intervenir de multiples aspects philosophiques, éthiques, conceptuels, économiques et d'application médicale pratique.

La meilleure approche est probablement de relier la notion de futilité (ou d'utilité) à des objectifs prédéterminés et de se poser trois questions : futile pour quoi ? futile pour qui ? futile comment ? le comment étant intimement lié au pour quoi et au pour qui.

\footnotetext{
A. Durocher $(\bowtie)$

Centre de réanimation-CHRU de Lille - Université de Lille

e-mail : alain.durocher@univ-lille2.fr
}

Cette approche rejoint la distinction proposée par Schneiderman entre effet et bénéfice, entre futilité quantitative et futilité qualitative [2].

\section{Futilité « quantitative » et essais thérapeutiques : pourquoi et comment?}

La futilité « quantitative » ou intrinsèque est l'incapacité d'un traitement à obtenir un résultat physiologique (futilité physiologique, par exemple l'incapacité d'un traitement à normaliser une glycémie ou une tension artérielle) et/ou l'incapacité d'un traitement à suppléer des défaillances d'organes. Les essais thérapeutiques bien menés permettent d'éviter cette futilité physiologique, mais la simple normalisation de variables physiologiques ou la correction de défaillances d'organes ne peut pas constituer l'objectif final de la réanimation. Ce ne sont que des objectifs secondaires, certes le plus souvent nécessaires, mais on peut très bien « mourir guéri » ou « guérir moribond »... L'objectif principal de la réanimation n'est pas là.

Les niveaux de preuve scientifique fournis par les essais thérapeutiques sont variables, d'une part selon leur design, d'autre part selon leurs validités interne et externe [3]. L'essai contrôlé, randomisé, prospectif, en aveugle et de préférence de supériorité (sur le principe du modèle expérimental) amène plus que tous les autres types d'essais un niveau de preuve scientifique important, permettant d'avoir la quasi-certitude de l'efficacité de la stratégie thérapeutique proposée (avec néanmoins un risque alpha accepté de se tromper de $5 \%$ ). Mais nombre d'études sont entachées d'un manque de puissance a posteriori.

Ce type d'étude n'est pas toujours réalisable pour des raisons éthiques, techniques, d'impossibilité d'aveugle, de prévalence de la pathologie, de question posée... et il faut alors se « contenter » d'études moins robustes, d'études cas- $t$ émoins, d'études de cohorte, de suivis de cas... qui n'amènent pas le même niveau de preuve scientifique.

Les essais doivent aussi prendre en compte la notion de futilité statistique, qui permet, par des analyses intermédiaires 
ad hoc et prédéfinies, de ne pas poursuivre un essai dont on peut prédire avant la fin des inclusions qu'il sera négatif et/ou entaché d'effets indésirables inacceptables. Plusieurs méthodes statistiques, initiées en cancérologie, sont maintenant à disposition et sont à utiliser en réanimation $[4,5]$.

Les réanimateurs doivent $s$ 'attacher à réaliser des essais méthodologiquement solides, notamment pour les stratégies émergentes, et à les publier, même et surtout s'ils sont négatifs, afin d'éviter cette futilité « quantitative».

Cependant, toutes les stratégies thérapeutiques et diagnostiques utilisées en réanimation ne sont pas passées par le filtre méthodologique de l'essai de type étude expérimentale pour les raisons citées ci-dessus ou pour des raisons historiques (la pénicilline par exemple). Pourtant, nombre de ces stratégies sont largement utilisées pour le plus grand bénéfice des patients sans pour autant être futiles.

Enfin, la négativité d'un essai ne peut être que la conséquence d'une grande variabilité pharmacogénomique de la population et de notre impossibilité actuelle de proposer en réanimation une médecine vraiment personnalisée et des traitements ciblés. Cela viendra.

\section{Futilité et recommandations de bonne pratique : pour quoi, pour qui et comment ?}

Pour aider les médecins à prendre la bonne décision et à éviter des traitements futiles, des processus d'élaboration de recommandations de bonne pratique permettent de recommander des stratégies thérapeutiques et diagnostiques en fonction du niveau de preuve scientifique fourni par la littérature [6]. Elles ont abouti à la notion d'evidence based medicine. Il ne faut néanmoins pas oublier que si les recommandations, même basées sur un niveau d'évidence scientifique, concernent certes une population de patients représentatifs d'un état pathologique donné, elles peuvent ne pas prendre en compte les particularités de la personne individuelle soignée ; en réanimation, les situations polypathologiques et de défaillance multiviscérale sont fréquentes, créent des situations complexes et singulières et rendent parfois difficilement applicables à la lettre les recommandations existantes. Ceci ne doit pas constituer pour autant une excuse à la futilité.

Dans nombre de cas, les recommandations proposées ne peuvent pas s'appuyer sur un niveau de preuve scientifique fort ; ce n'est pas pour autant que la recommandation proposée est futile, car elle a pris en compte d'autres éléments (études de faible niveau de preuve, avis d'experts, état des pratiques) et fait l'objet d'un consensus professionnel. Il ne faut pas confondre futilité et absence d'evidence-based, même si tous les efforts doivent être faits pour sortir de ces zones d'ombre scientifique et aboutir à des recommandations basées sur un niveau de preuve scientifique.
Ce sont ces recommandations reposant sur un consensus professionnel prédominant, pour lesquelles il est important que les professionnels de santé prennent le soin d'associer, lors de l'élaboration de ces recommandations, des représentants de la société civile, des patients et parfois des familles de patients. Les preuves scientifiques faibles ou absentes sont alors confrontées à des avis non médicaux, sociaux, sociétaux, environnementaux, juridiques...

En réanimation, malgré les énormes progrès faits ces dernières décennies sur les plans physiopathologique, diagnostique et thérapeutique, malgré la mise à disposition des réanimateurs de nouvelles techniques et de nouveaux matériels (dont l'intérêt réel et le service médical rendu ne sont pas toujours prouvés), la décision médicale reste encore bien souvent une décision en situation d'incertitude. D'autre part, ces mêmes avancées médicales et technologiques ont un coût et amènent à se poser la question de leur réelle utilité ou futilité. Ainsi, des travaux ont montré que des traitements jugés inutiles (ou futiles) pouvaient représenter 3,5\% des coûts de prise en charge des patients d'une unité de réanimation adulte [7] et $4,5 \%$ de ceux d'une unité de réanimation néo-natale [8].

Enfin, les scores de gravité ou de défaillances viscérales largement utilisés en réanimation ne sont que des outils populationnels de nature statistique peu discriminants et statiques au regard des progrès des prises en charge : ils ne peuvent en aucun cas être utilisés comme outil d'aide à une décision individuelle chez des patients évolutifs dans le temps.

C'est alors que la notion de futilité (ou d'utilité) quitte la sphère quantitative et s'avance vers des terrains plus meubles et la notion de futilité (ou d'utilité) qualitative.

\section{Futilité, déontologie et éthique : pour qui et comment ?}

Le terme qualitatif renvoie à la notion de qualité : qualité voulue et rendue (par le réanimateur) ou qualité attendue et perçue (par le patient et ses proches).

La qualité voulue et rendue fait partie de la démarche naturelle du réanimateur : elle est la traduction du Code de déontologie médicale « d'assurer personnellement au patient des soins (...) fondés sur les données acquises de la science » (art. 32 du Code de déontologie médicale) [9], tout en tenant compte des avantages, des inconvénients et des conséquences des différentes investigations et thérapeutiques possibles (art. $8 \mathrm{du}$ Code de déontologie médicale). Cet article 8 aborde d'ailleurs la notion de futilité, puisqu'il précise que les prescriptions et les actes doivent être limités à ce qui est nécessaire à la qualité, à la sécurité et à l'efficacité des soins.

Le Code de déontologie rejoint ainsi les principes de l'éthique, notamment les principes de bienfaisance et de non-nuisance. 
La qualité attendue et perçue est la traduction de l'attente en termes d'objectifs de « quelle vie et comment vivre? »du patient et/ou de ses proches et de leur satisfaction. L'attente du patient ou de ses proches est l'expression du principe d'autonomie et du choix en tant que personne et pas seulement en tant que malade réanimé ou risquant d'être réanimé. Il s'agit d'un principe d'inviolabilité de la personne et de la prépondérance de sa volonté personnelle. Or ce choix n'est pas uniquement de (sur)vivre, de ne pas être malade, mais d'être en bonne santé. La santé, définie par l'OMS, est un ensemble de conditions qui permettent l'épanouissement physiologique, physique, psychologique, esthétique, social, de l'homme sans s'arrêter à la seule notion d'absence de handicap ou de maladie. La notion de santé attendue et perçue est donc multifactorielle et le poids accordé par chacun à l'un des composants de cette "bonne santé " est très variable.

Les possibilités actuelles de la réanimation en matière de suppléance d'organes et les progrès technologiques permettent théoriquement de maintenir en vie (ou en survie) de façon prolongée et posent obligatoirement la question du bénéfice potentiel pour le patient en termes d'objectifs de santé et de qualité de (sur)vie .Cette réflexion a amené à des recommandations de limitations de traitement, d'arrêts de traitement, de non-admission en réanimation, où le médecin réanimateur ne peut plus et ne doit plus être seul à décider et doit parfois proposer collégialement des options autres que la vie [1], notamment quand il se trouve dans une zone grise d'indécision [10]. Être réanimateur, c'est lutter pour la vie mais c'est aussi assurer dans tous les cas le confort du patient, même en l'absence d'espoir déraisonnable de survie ou de non-atteinte des objectifs personnels du patient. C'est conjuguer evidence based medicine et preference-based medicine.

\section{Références}

1. Actualisation des Recommandations de la Société de Réanimation de Langue Française (2010) Limitation et arrêt des traitements en réanimation adulte. Réanimation 19:679-98

2. Schneiderman LJ, Jecker NS, Jonsen AR (1990) Medical futility: its meaning and ethical implications. Ann Intern Med 112:949-54

3. Association Pédagogique Nationale pour l'Enseignement de la Thérapeutique (APNET) (2014) Lecture critique d'un article médical. 300 pages, Éditions Medline

4. Kramar A, Paoletti X (2007) Analyses intermédiaires. Bull Cancer 94:965-74

5. Snappin S, Chen MG, Jiang Q, Koutsoukos T (2006) Pharmaceut. Statist. 5:273-81

6. Haute Autorité de Santé (2013) Niveau de preuve et gradation des recommandations de bonne pratique. 90 pages, www.hassante.fr

7. Huynh TN, Kleerup EC, Wiley JF, et al (2013) The frequency and cost of treatment perceived to be futile in critical care. JAMA Intern Med 173:1887-94

8. Meadow X, Cohen-Cutler S, Spelke B, et al (2012) The prediction and cost of futility in the NICU. Acta pediatr 4:397-402

9. Conseil National de l'Ordre des Médecins (2012) Code de déontologie médicale. www.conseil-national.medecin.fr

10. Misak CJ, Phil D, White DB, Truog RD (2014) Medical futility: a new look at an old problem. Chest 146:1667-72 